\title{
Expression profile of apoptotic and proliferative proteins in hypoxic HUVEC treated with statins
}

\author{
XIAOCHEN LI, XIANSHENG LIU, YONGJIAN XU, YUANZHOU HE, JIN LIU and MIN XIE \\ Department of Respiratory and Critical Care Medicine, Tongji Hospital, Tongji Medical College, \\ Huazhong University of Science and Technology, Wuhan, Hubei, P.R. China
}

Received September 20, 2014; Accepted November 6, 2014

DOI: $10.3892 /$ ijo.2014.2780

\begin{abstract}
Vascular endothelial hyperproliferation is involved in the pathophysiological process of angiogenesis, which is indispensable for tumor growth and spread in hypoxic adaptation. There is increasing evidence indicating that statins have potential anti-angiogenesis benefits. However, the intracellular signaling mechanism underlying the effect of statins in vascular endothelial cells is undefined. The present study was conducted to investigate the effect of fluvastatin on cell proliferation and apoptosis in normoxic and hypoxic human umbilical vein endothelial cells (HUVEC). Flow cytometric analyses revealed that statins reversed hypoxia-induced cell proliferation by slowing down $\mathrm{G}_{1}$ to $\mathrm{S}$ transition and inducing cell apoptosis. To get further insights into the downstream effects of statins, we measured the expression of various apoptosis-associated proteins in hypoxic HUVEC using human apoptosis antibody array. The results suggested that cell apoptosis was accompanied by upregulation of caspase-3, p27, IGFBP- 6 and a decrease of bcl-2, survivin levels. Subsequent studies confirmed the results of array and demonstrated that fluvastatin activated mitochondrial apoptosis through enhancing bax/bcl-2 ratio, releasing cytochrome $c$, in turn activating caspase- 9 and caspase-3, and eventually cleaving PARP. Further experiments showed that inhibition of cell proliferation by fluvastatin was associated with elevated IGFBP-6, p27, p53 levels and reduced survivin, cyclin B1, cyclin D1 and VEGF expression. Taken together, fluvastatin suppressed cell proliferation and induced apoptosis of HUVEC in hypoxia via multiple signaling pathways, providing a theoretical basis for statins in the therapy of cancer.
\end{abstract}

Correspondence to: Professor Xiansheng Liu, Department of Respiratory Diseases, Tongji Hospital, Key Lab of Pulmonary Diseases of Health Ministry, Tongji Medical College, Huazhong University of Science and Technology, 1095 Jiefang Avenue, Wuhan, Hubei 430030, P.R. China

E-mail: doctorliu69@126.com

Key words: statins, hypoxia, mitochondrial apoptosis, cell cycle, endothelial cells

\section{Introduction}

Angiogenesis is a physiological process that new blood vessels grow from existing microvasculature in ischemic tissues. Angiogenesis plays a critical role in tumor progression by supplying nutrients, oxygen and removing metabolic waste for tumor growth and metastasis (1). Microvessel density (MVD), reflecting angiogenesis, has been proven to be an important prognostic factor in a wide range of tumors (2-4).

Endothelial hyperproliferation is a critical step in the process of angiogenesis and is triggered by a diverse of biochemical and microenvironment factors. Hypoxia occurs as a result of an imbalance between oxygen supply and consumption due to exponential cellular proliferation in tumor. In order to adapt to a hostile environment, hypoxia changes the phenotype of endothelial cells by activating a series of genes, including vascular endothelial growth factor (VEGF), platelet-derived growth factor-B (PDGF-B) and transforming growth factor (TGF) (5). Considering that the activation of vascular endothelial cell proliferation and resistance to apoptosis are vital characteristics of angiogenesis, induction of cell apoptosis and cell cycle arrest may be a promising treatment strategy for cancer.

Statins, also known as 3-hydroxy-3-methyl-glutaryl-CoA (HMG-CoA) reductase inhibitors, are a class of compounds used to lower cholesterol and its isoprenoid intermediates, accompanied by elevation of HDL-cholesterol. Both HDL particles and lower cholesterol level are protective factors for blood vessels. A finding shows that cholesterol efflux from endothelial cells to high-density lipoprotein (HDL) regulates angiogenesis, which implies the key role of cholesterol metabolism in proper angiogenesis (6). Nevertheless, the non-cholesterol dependent effect of statins on endothelial function, vascular inflammation, cell proliferation, immunomodulation (7) and oxidation (8) is of even greater concern.

A substantial body of data suggests that statins are able to prevent angiogenesis by modulating endothelial cells. Fluvastatin has apoptosis-inducing effect in endothelial cells by the activation of caspase-3 and the presence of extensive DNA fragmentation (9). Pravastatin inhibits endothelial proliferation through inducing $\mathrm{G}_{1}$ arrest and decreasing cyclin D1, cyclin $\mathrm{E}$ and cyclin-dependent kinase 2 expression (10). Statins exert a biphasic dose-dependent effect on angiogenesis by stimulating angiogenesis at low concentrations and inducing endothelial apoptosis with high-dose treatment (11). 
Vascular endothelial cell is a key component in angiogenesis and is also an attractive target of statins. Notably, so far the endothelial effects of statins in hypoxia have not been clearly elucidated. Therefore, we conducted the present study to test the effects of fluvastatin on cell growth in hypoxic human umbilical vein endothelial cells (HUVEC) and identify the underlying mechanisms, providing evidence for future development in cancer treatment.

\section{Materials and methods}

Reagents. Fluvastatin was purchased from Sigma-Aldrich Inc. (St. Louis, MO, USA). Stock solution of $10 \mathrm{mg} / \mathrm{ml}$ was prepared by dissolving fluvastatin powder in dimethyl sulfoxide (DMSO) (Invitrogen, Carlsbad, CA, USA). The previous stock solution $(2 \mu \mathrm{l})$ was diluted with $98 \mu \mathrm{l}$ of phosphate-buffer solution (PBS) to give a fresh secondary solution $(0.2 \mathrm{mg} / \mathrm{ml})$ each time it was required. Working solutions of different concentrations were obtained directly in the culture media and DMSO controls were used in the study at a range of concentrations between $1.355 \times 10^{-6}$ and $6.938 \times 10^{-4}(\mathrm{vol} / \mathrm{vol})$ to overcome the possible interference of DMSO.

Cells and cell culture. HUVEC were obtained from the American Type Culture Collection (ATCC, Manassas, VA, USA) and cultured in F-12K medium (Gibco, Grand Island, NY, USA) supplemented with $10 \%$ ( $\mathrm{vol} / \mathrm{vol}$ ) heat-inactivated fetal bovine serum (Gibco) and $0.05 \mathrm{mg} / \mathrm{ml}$ endothelial cell growth supplement (ECGS). Cell cultures were maintained at $37^{\circ} \mathrm{C}$ in a humidified atmosphere incubator (Hera cell 150; Heraeus, Langenese, Germany) of $5 \% \mathrm{CO}_{2}$ in air. Induction of hypoxic condition was performed in a modular incubator (Galaxy R, RS Bitotech, Alloa, UK) flushed with $1 \% \mathrm{O}_{2}, 5 \% \mathrm{CO}_{2}$ and $94 \% \mathrm{~N}_{2}$.

Cell viability assay. HUVEC were seeded in 96-well plates at a density of $2 \times 10^{3} /$ well. Cells were exposed to normoxia or hypoxia and treated with fluvastatin or DMSO at different concentrations of $0.125,0.25,0.5,1,2,4,8$ and $16 \mu \mathrm{M}$ for $48 \mathrm{~h}$. Cell Counting Kit-8 (CCK-8) (Dojindo Laboratories, Tokyo, Japan) was used to measure cell viability. Cells were incubated with $10 \mu \mathrm{lCCK}-8$ solution at $37^{\circ} \mathrm{C}$ for $4 \mathrm{~h}$ following the manufacturer's instructions. Optical density (OD) values at $450 \mathrm{~nm}$ were obtained using an ELx800 Universal Microplate Reader (BioTek Instruments, Inc., Winooski, VT, USA).

Cell apoptosis assay. Cell apoptosis was quantified using the Annexin V/propidium iodide (PI) detection kit (Beyotime, Shanghai, China) and analyzed by flow cytometry. Cells ( $2 \times 10^{5} /$ well) were plated in 6 -well dishes and incubated with $0.03125,0.0625,0.125,0.25,0.5,1,2,4,8$ and $16 \mu \mathrm{M}$ fluvastatin or DMSO exposed to hypoxia. After 48-h treatment, collected cells were incubated in $400 \mu \mathrm{l}$ binding buffer with $5 \mu \mathrm{l}$ Annexin V-FITC and $5 \mu \mathrm{l}$ PI in dark for $15 \mathrm{~min}$ at room temperature (RT). The cells were analyzed by flow cytometry (BD Biosciences, San Jose, CA, USA) to identify the early apoptotic (Annexin V positive and PI negative) and late apoptotic (Annexin $\mathrm{V}$ positive and PI positive) cells.

Cell cycle assay. HUVEC were harvested and fixed in $70 \%$ cold ethanol at $4^{\circ} \mathrm{C}$ overnight. Cells were washed with cold
PBS and resuspended in $400 \mu \mathrm{l}$ staining buffer with $25 \mu \mathrm{l} \mathrm{PI}$ solution and $10 \mu \mathrm{l}$ RNase A (Beyotime) at $37^{\circ} \mathrm{C}$ in a water bath. Flow cytometry data were analyzed with BD FACSDiva 6.1.

Human apoptosis antibody array. The expression of apoptosis-related proteins was detected using a human apoptosis antibody array kit (RayBiotech Inc., Norcross, GA, USA) according to the protocols. This glass chip contains duplicate spots of 43 apoptosis-related proteins. Briefly, the slides were incubated with blocking buffer at RT for $30 \mathrm{~min}$. Lysates of HUVEC treated with fluvastatin or DMSO were then added and kept at $4{ }^{\circ} \mathrm{C}$ overnight. After thorough rinsing, the chip was incubated with biotin-conjugated antibodies at RT for $2 \mathrm{~h}$ followed by addition of streptavidin. The chip was scanned using the Axon GenePix laser scanner (Molecular Devices Corp., Union City, CA, USA) and the images were quantified by GenePix Pro 6.0 software.

Real-time PCR. Total RNA was extracted using TRIzol (Takara, Dalian, China). The semi-quantitative assessment of cellular mRNA expression were performed using reverse transcription polymerase chain reaction (RT-PCR) kit (Takara) and SYBR Green real-time quantitative PCR (qPCR) kit (Takara). The signals were collected and analyzed by ABI Prism Fast 7500 system (Applied Biosystems, Foster City, CA, USA). The amplification conditions were: preheat at $95^{\circ} \mathrm{C}$ for $30 \mathrm{sec}, 40$ cycles of $95^{\circ} \mathrm{C}$ for $5 \mathrm{sec}, 60^{\circ} \mathrm{C}$ for $34 \mathrm{sec}$ followed by $95^{\circ} \mathrm{C}$ for $15 \mathrm{sec}$ and $60^{\circ} \mathrm{C}$ for $1 \mathrm{~min}$. Primers were: $\beta$-actin F-5'-AGCGAGCATCCCCCAAAGTT-3' and R-5'-GGG CACGAAGGCTCATCATT-3'; IGFBP-6 F-5'-CATGCCGT AGACATCTGGAC-3' and R-5'-GGTAGAAGCCTCGAT GGTCA-3'; BAX F-5'-AAGCTGAGCGAGTGTCTCAAG-3' and R-5'-CAAAGTAGAAAAGGGCGACAAC-3'; BCL-2 F-5'-ATGTGTGTGGAGAGCGTCAAC-3' and R-5'-AGAGA CAGCCAGGAGAAATCAAAC-3'; p27 F-5'-CGGGACTT GGAGAAGCACT-3' and R-5'-AGTAGAACTCGGGCAA GCTG-3'; p53 F-5'-CCCCTCCTGGCCCCTGTCATCTTC-3' and R-5'-GCAGCGCCTCACAACCTCCGTCAT-3'; CCNB F-5'-CTGGATAATGGTGAATGGACAC-3' and R-5'-CGAT GTGGCATACTTGTTCTTG-3'; CCND F-5'-CGGAGGAGA ACAAACAGATCAT-3' and R-5'-AGGCGGTAGTAGGAC AGGAAGT-3'; BIRC F-5'-CACCGCATCTCTACATTCAA GA-3' and R-5'-CAAGTCTGGCTCGTTCTCAGT-3'; VEGF F-5'-TTCTGAGTTGCCCAGGAGAC-3' and R-5'-TGGTTT CAATGGTGTGAGGA-3.

Western blot analysis. Cell lysates of HUVEC treated with fluvastatin or DMSO under hypoxia were analyzed by western blotting using antibodies to glyceraldehyde-3-phosphate dehydrogenase (GAPDH), bax, bcl-2, caspase-9, caspase-3, cytochrome $c, \mathrm{p} 53$, cyclin B1, cyclin D1, survivin and VEGF. The antibodies listed above were purchased from Proteintech Group (1:1,000 dilution for western blots). In addition, the antibodies to voltage-dependent anion-selective channel (VDAC), poly(ADP-ribose) polymerase (PARP), insulin-like growth factor binding protein-6 (IGFBP-6), p27 were obtained from Abcam Inc. (1:1,000 dilution for western blots). Mitochondrial proteins were extracted using cell mitochondria isolation kit (Beyotime). Blots were reprobed with GAPDH and VDAC to confirm equal loading of cell lysate and mitochondrial 

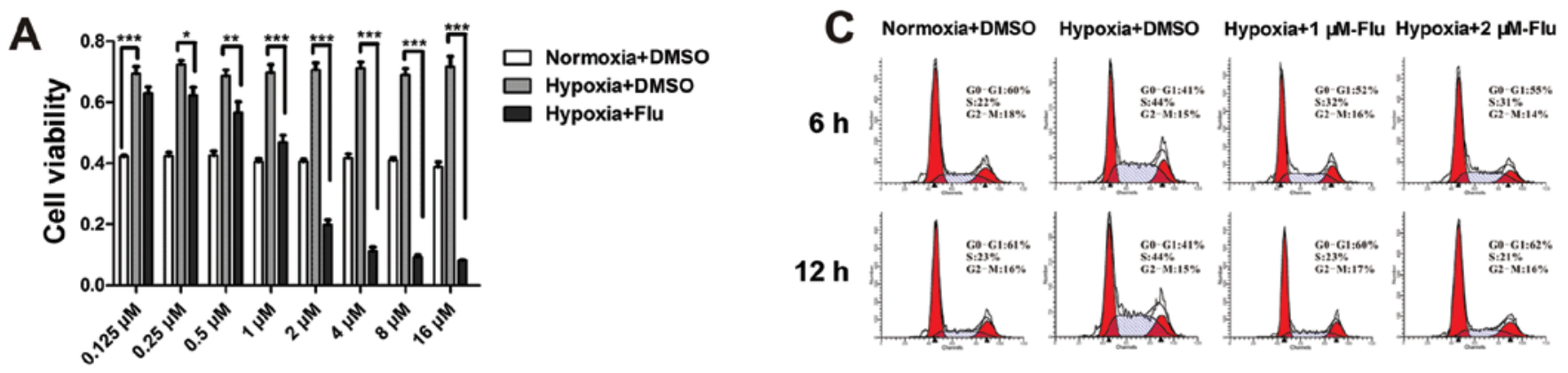

B

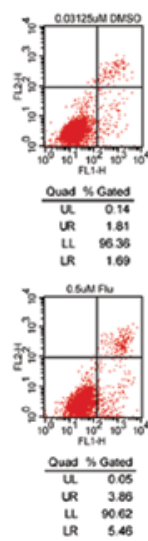

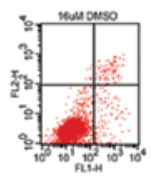
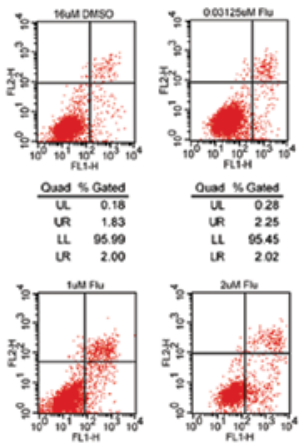

\begin{tabular}{cc} 
Onod $\$$ Goled \\
\hline$u$ & 0.92 \\
UR & 10.96 \\
$u$ & 81.8 \\
UR & 7.14
\end{tabular}
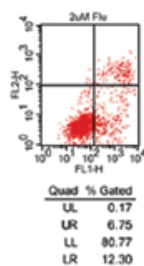
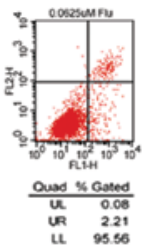

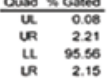

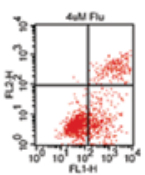

\begin{tabular}{ccc} 
oud 5 couted \\
\hline$u$ & 0.12 \\
$U R$ & 14.37 \\
$u$ & 83.81 \\
$u$ & 21.70
\end{tabular}
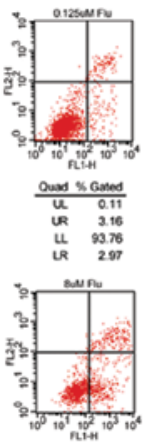

\begin{tabular}{cc} 
Ouad & ranis \\
\hline$U$ & 0.55 \\
UR & 14.79 \\
$u$ & 60.67 \\
UR & 2396
\end{tabular}
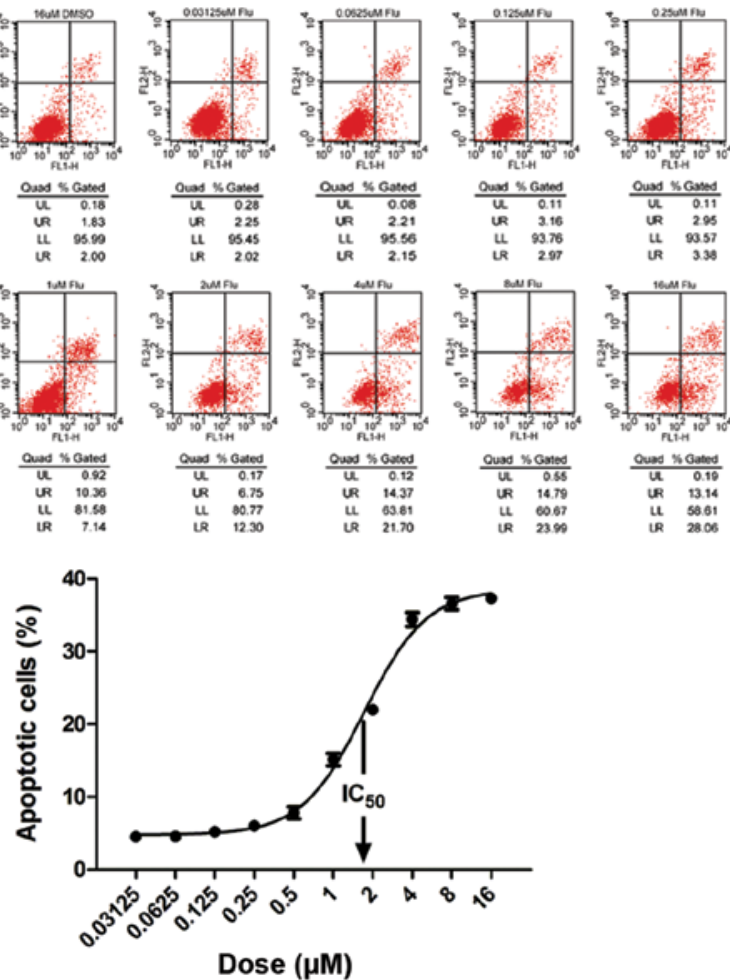
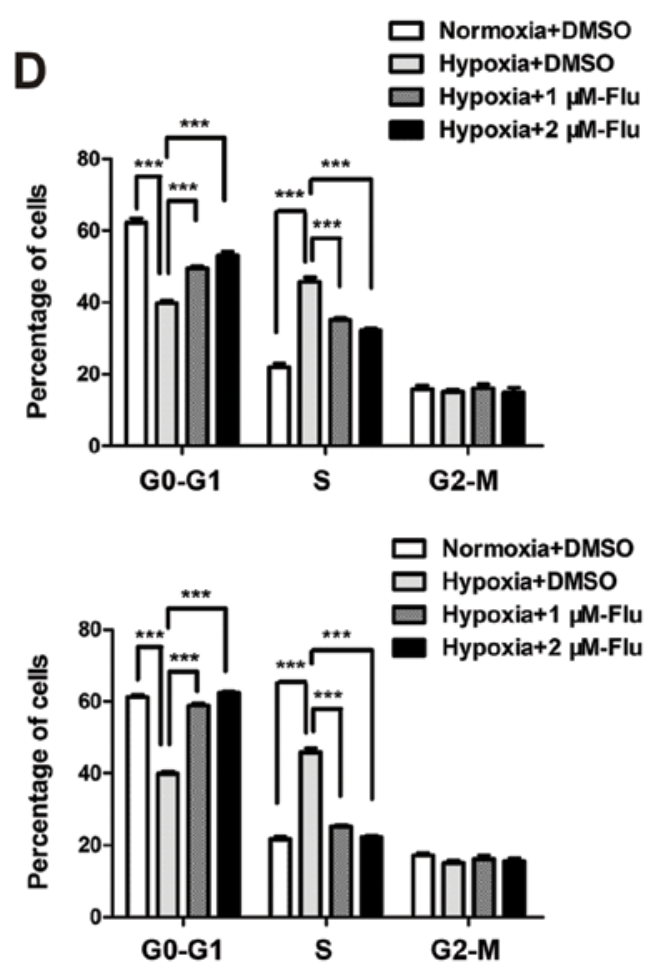

Figure 1. Fluvastatin reverses proliferation-enhancing effect of hypoxia on HUVEC and induces cell apoptosis. HUVEC were treated with different concentrations of fluvastatin or DMSO exposed to hypoxia $\left(1 \% \mathrm{O}_{2}\right)$ or normoxia $\left(20 \% \mathrm{O}_{2}\right)$. (A) CCK-8 tests were performed to measure cell viability after 48 -h treatment and OD values were analyzed. (B) Cell apoptosis was determined by Annexin V/propidium iodide assay in HUVEC with fluvastatin treatment and hypoxia exposure for $48 \mathrm{~h}$. Horizontal axis represents intensity of staining for Annexin V and vertical axis indicates the detection of cells with propidium iodide (PI). The apoptotic cells were calculated as the sum of both early apoptosis (Annexin V positive and PI negative) and late apoptosis (Annexin V positive and PI positive). (C) PI staining was carried out to evaluate the cell cycle in HUVEC. Cells were treated with fluvastatin for 6 and $12 \mathrm{~h}$ followed by PI staining and quantification of cell cycle at 6 and $12 \mathrm{~h}$ are shown in the upper and lower figure (D). Data are shown as mean $\pm \mathrm{SEM} ; \mathrm{n}=4$. $^{*} \mathrm{P}<0.05,{ }^{* *} \mathrm{P}<0.01,{ }^{* * *} \mathrm{P}<0.001$. Flu, fluvastatin.

proteins. The intensity of protein bands was quantified by Image J and normalized to GAPDH and VDAC in the analyses.

Statistical analysis. Statistical analysis was performed in GraphPad Prism 5.0 software (GraphPad Software Inc., San Diego, CA, USA). Comparison between two groups was analyzed by the Student's t-test and multi-group analysis of variances was carried out by two-way ANOVA with Bonferroni post test. Apoptotic cells following fluvastatin treatment increased with different concentrations. $\mathrm{IC}_{50}$ was analyzed using a non-linear regression of log (inhibitor) vs. response (variable slope). All experiments were repeated at least four times and the data are expressed as the mean \pm SEM. $\mathrm{P}<0.05$ is considered to be statistically significant $\left({ }^{*} \mathrm{P}<0.05,{ }^{* *} \mathrm{P}<0.01\right.$ or ${ }^{* * *} \mathrm{P}<0.001$, respectively, in the figures).

\section{Results}

Fluvastatin inhibits hypoxia-induced HUVEC proliferation and accelerates apoptosis. The effects of fluvastatin on cell viability, apoptosis and cell cycle were evaluated by the CCK-8 test, Annexin V/PI detection and PI staining. Hypoxia significantly increased cell viability of HUVEC and fluvastatin completely reverse the enhancement compared with DMSO controls. The inhibition rate rose with the increasing concentrations ranging from 0.125 to $16 \mu \mathrm{M}$ (Fig. 1A).

To investigate the apoptosis-inducing effect and the half maximal inhibitory concentration $\left(\mathrm{IC}_{50}\right)$ of statin, HUVEC were incubated with fluvastatin at 10 concentrations ranging from 0.03125 to $16 \mu \mathrm{M}$ under hypoxia for $48 \mathrm{~h}$. Apoptosis rate was assessed by the sum percentage of early and late apoptotic 
A

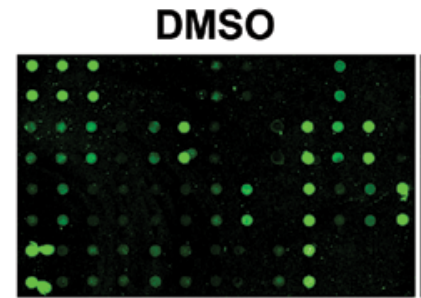

$1 \mu \mathrm{M}-\mathrm{Flu}$

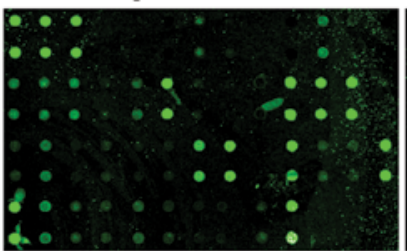

$2 \mu \mathrm{M}$-Flu

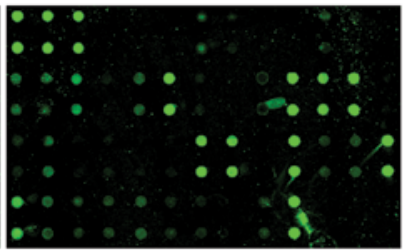

B

\begin{tabular}{|c|c|c|c|c|c|c|c|c|c|c|c|c|c|}
\hline & $A$ & $B$ & $B$ & 0 & $E$ & $F$ & $G$ & $\mathrm{H}$ & 1 & $\sqrt{J}$ & K & L & $M$ \\
\hline 1 & Pos 1 & Pos 2 & Pos 3 & $\mathrm{Neg}$ & $\mathrm{Neg}$ & bad & bax & $b c 1.2$ & $b c=w$ & $B I D$ & BIM & Caspase3 & caspase8 \\
\hline 2 & Pos 1 & Pos 2 & Pos 3 & $\mathrm{Neg}$ & $\mathrm{Neq}$ & bad & bax & $b c 0.2$ & bolw w & $B I D$ & BIM & Caspase3 & caspase8 \\
\hline 3 & $\mathrm{CD} 40$ & CDAOL & CIAP.2 & croc & DR6 & Fas & FasL & neg & HSP27 & HSPGO & HSP70 & HTRA & $|G F \cdot|$ \\
\hline 4 & $\mathrm{CO} 40$ & CD4OL & CIAP.2 & Croc & DA6 & Fas & FasL & neg & HSP27 & HSP60 & HSP70 & HTRA & IGF. \\
\hline 5 & $\mid G F \cdot \|$ & IGFBP.1 & IGFBP.2 & IGFBP.3 & IGFBP.4 & IGFBP.5 & IGFBP.6 & IGF.1SR & Ivin & $p 21$ & p27 & 053 & SMAC \\
\hline 6 & IGF. II & IGFBP.1 & IGFBP.2 & IGFBP.3 & IGFBP.4 & IGFBP.5 & IGFBP.6 & IGF.18R & Ivin & $p 21$ & .227 & .053 & SMAC \\
\hline 9 & Survivin & $S T$ STF.R1 & $\overline{S T N F \cdot R 2}$ & TNF-alpha & TNF-beta & TRAILR:1 & $\overline{\text { TRAILR:2 }}$ & TRAILR:3 & $\overline{\text { TRAILR.4 }}$ & XIAP & $\mathrm{Neg}$ & $\mathrm{Neg}$ & $\mathrm{Neg}$ \\
\hline 8 & Survivin & STNF.R1 & STNF-R2 & TNF-alpha & TNF-beta & TRAILR:1 & TRAILR.2 & TRAILP:3 & TRAILR-4 & XIAP & $\mathrm{Neg}$ & $\mathrm{Neg}$ & Neg \\
\hline
\end{tabular}

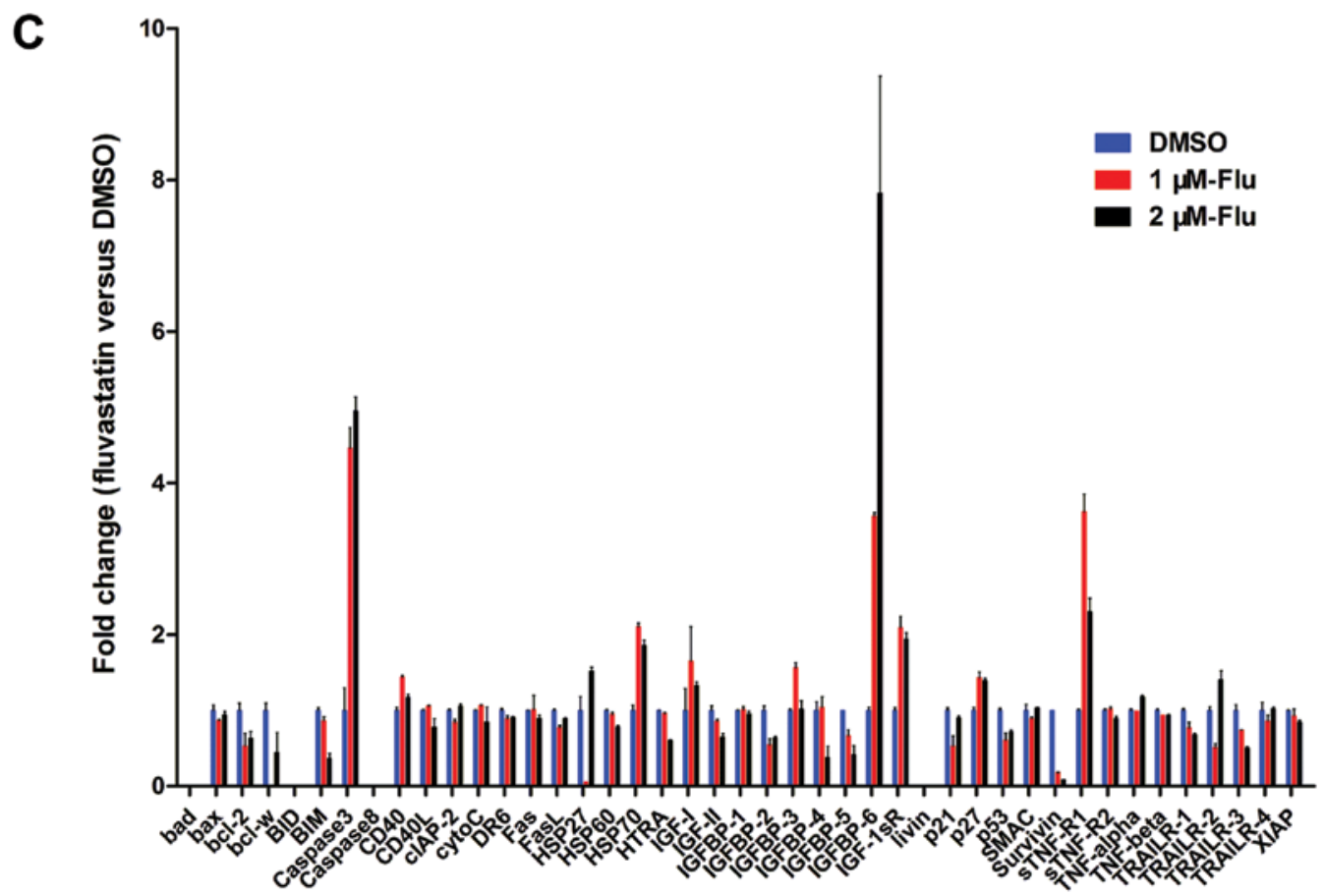

Figure 2. Apoptosis array analysis. (A) Nine subarrays were probed to identify apoptotic proteins in hypoxic HUVEC treated with DMSO, 1 and $2 \mu \mathrm{M}$ fluvastatin. Three representative scanned images are shown. (B) Forty-three targets detected in the chips with a one-to-one correspondence. (C) The levels of proteins were quantified and data were expressed as fold of DMSO controls. Data are shown as mean \pm SEM. Flu, fluvastatin.

cells. The data showed that the maximum apoptotic effect was $\sim 40 \%$ and $\mathrm{IC}_{50}$ value was between 1 and $2 \mu \mathrm{M}$ (Fig. 1B). Therefore, 1 and $2 \mu \mathrm{M}$ were chosen as concentrations of fluvastatin in the following experiments. It is worth noting that necrotic cells (Annexin $\mathrm{V}$ negative and PI positive) were not observed in the experiment (Fig. 1B).

The effect of fluvastatin on cell cycle of HUVEC was expressed by the percentage of cells in $\mathrm{G}_{0}-\mathrm{G}_{1}, \mathrm{~S}, \mathrm{G}_{2}-\mathrm{M}$ phase. A significant increase in $\mathrm{S}$-phase cells was observed following hypoxia. Fluvastatin reversed the transition of $\mathrm{G}_{1} / \mathrm{S}$ compared with DMSO controls in a time-dependent manner (Fig. 1C and $D)$. The number of $G_{2}-M$ phase cells was not affected after $12 \mathrm{~h}$ of fluvastatin treatment.
The results suggest that fluvastatin mitigates hypoxiainduced abnormal proliferation of HUVEC through inducing cell apoptosis and preventing cell cycle progression.

Analysis of human apoptosis antibody array. To make clear the molecular profile of decreased cell growth, human apoptosis antibody array was exploited to analyze the apoptosis-related proteins expression in HUVEC with fluvastatin treatment at 1 and $2 \mu \mathrm{M}$ exposed to hypoxia for $48 \mathrm{~h}$ (Fig. 2A and B). The data showed that there is an obvious increase of caspase-3, heat shock protein (HSP) 70, IGFBP-6, IGF-1sR, p27 and soluble tumor necrosis factor receptor-1 (sTNF-R1) levels while bcl-2, bim, p53 and survivin were downregulated (Fig. 2C). 


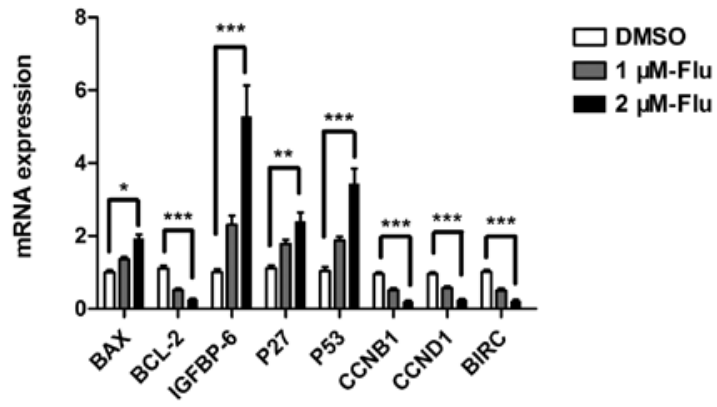

Figure 3. The mRNA expression of cell growth-associated factors following fluvastatin treatment in hypoxia for $12 \mathrm{~h}$. Data are shown as mean $\pm \mathrm{SEM}$; $\mathrm{n}=4 .{ }^{*} \mathrm{P}<0.05,{ }^{, * *} \mathrm{P}<0.01,{ }^{* * *} \mathrm{P}<0.001$. Flu, fluvastatin.
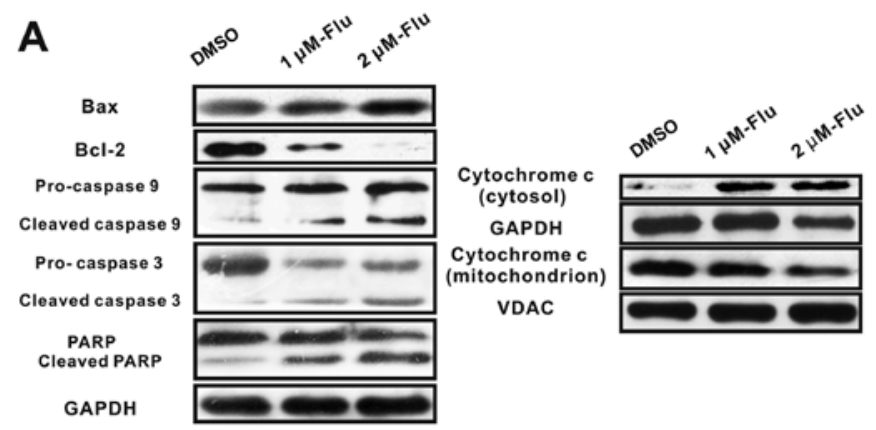

B

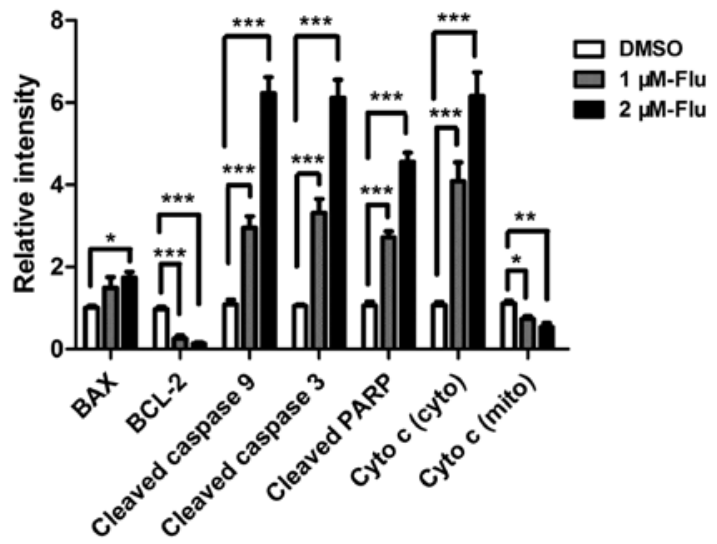

Figure 4. Fluvastatin activates mitochondrial apoptosis pathway in HUVEC. (A) Representative western blotting and (B) quantification of proteins in HUVEC treated with fluvastatin or DMSO exposed to hypoxia for $48 \mathrm{~h}$ are shown. In the cytochrome $c$ measurement, mitochondrial protein and cytosolic fraction were separately isolated from HUVEC and VDAC was used as internal reference for cytochrome $c$ in the mitochondrion. Data are shown as mean \pm SEM; $n=4 .{ }^{*} \mathrm{P}<0.05,{ }^{* *} \mathrm{P}<0.01,{ }^{* * *} \mathrm{P}<0.001$. Flu, fluvastatin; VDAC, voltage-dependent anion-selective channel; cyto, cytosol; mito, mitochondrion.

Gene expression of apoptotic mediators and proliferative proteins. The regulation of cell cycle is a key element controlling cell proliferation. Cell cycle checkpoints are responsible for ensuring cell division in a steady and correct way. Cyclin B1 and cyclin D1 are, respectively, required for $\mathrm{G}_{2} / \mathrm{M}$ and $\mathrm{G}_{1} / \mathrm{S}$ transition, both of which were explored in the present study. According to the results of antibody array and cell cycle analysis, we performed real-time PCR to detect mRNA expression of bax, bcl-2, bim, HSP 70, IGFBP-6, IGF-1sR, p27, p53, sTNF-R1, cyclin B1, cyclin D1 and survivin. The results confirmed gene expression changes of bax, bcl-2, IGFBP-6,
A

IGFBP 6

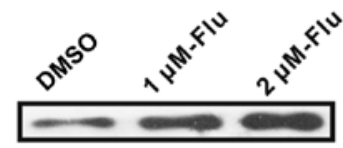

P53

Cyclin B1

Cyclin D1

Survivin

GAPDH

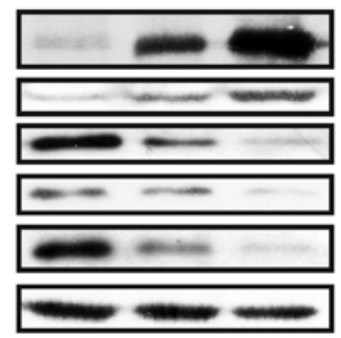

B

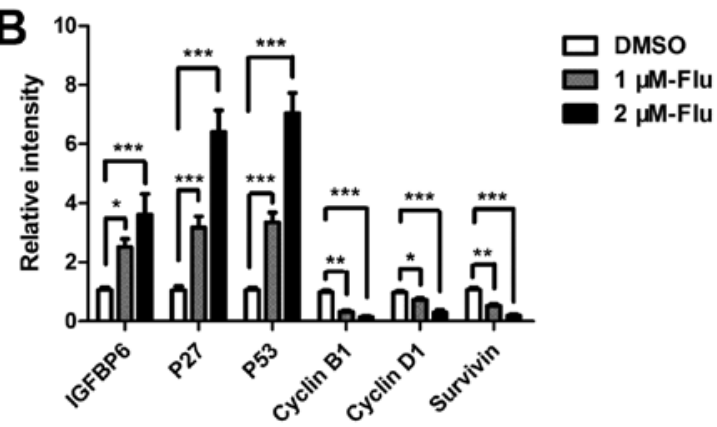

Figure 5. Levels of the proliferation associated proteins. Western blotting was carried out in hypoxic HUEVC treated with fluvastatin or DMSO. (A) The representative images and $(\mathrm{B})$ quantification analysis are shown. Data are shown as mean \pm SEM; $n=4 .{ }^{*} \mathrm{P}<0.05,{ }^{* *} \mathrm{P}<0.01,{ }^{* * *} \mathrm{P}<0.001$. Flu, fluvastatin.

p27, cyclin B1, cyclin D1, survivin and showed a significant upregulation of p53 (Fig. 3).

Fluvastatin activates mitochondrial pathway of apoptosis. Mitochondrial pathway is initiated by the altered expression of pro-apoptotic molecules and anti-apoptotic proteins. The increase of bax $/ \mathrm{bcl}-2$ ratio promotes the release of mitochondrial cytochrome $c$ which binds to apoptotic protease activating factor-1 (Apaf-1), ATP and pro-caspase-9 to form a complex, called an apoptosome. The apoptosome cleaves caspase-9, and caspase-3 then activating PARP, leading to cell death (12). Because bax, bcl-2 and caspase-3 were mediated by fluvastatin in protein array and real-time PCR analyses, we next examined whether fluvastatin treatment modulated bax, bcl-2, cytochrome $c$, caspase-9, caspase-3, and PARP expression in HUVEC using western blotting. As expected, the protein level of bax was augmented and bcl-2 was significantly inhibited, implying the activation of mitochondrial apoptosis. Cytochrome $c$ was released from mitochondria and cleaved caspase-3, caspase-9 and PARP were induced, indicating that fluvastatin induced cell apoptosis mainly by the mitochondrial pathway (Fig. 4).

Regulation of proteins involved in the inhibition of cell proliferation. Analyses of real-time PCR suggested that proliferation-related proteins IGFBP-6, cyclin B1, cyclin D1, p27, p53 and survivin were involved in the regulation of cell growth following fluvastatin treatment. Therefore, the levels of proteins were measured by western blotting and the results showed that fluvastatin significantly enhanced IGFBP-6, p27 and p53 and reduced cyclin B1, cyclin D1 and survivin (Fig. 5). 
A

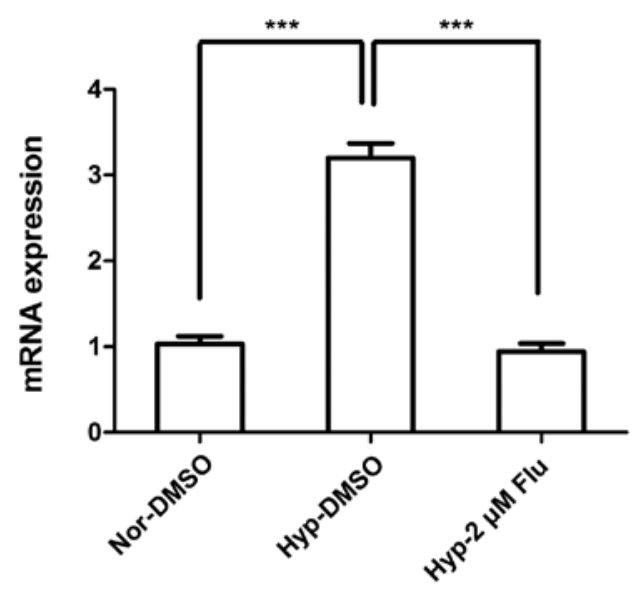

B

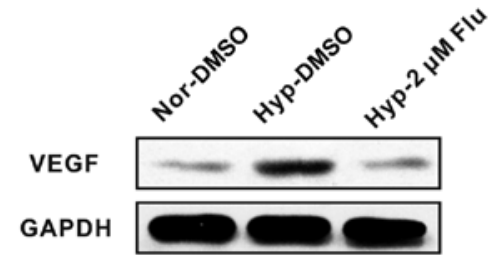

C

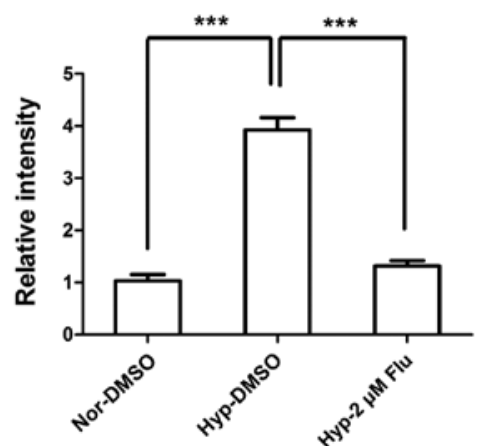

Figure 6. Hypoxia enhances VEGF expression and fluvastatin suppresses the increase of VEGF in HUVEC. (A) The mRNA and protein (B and C) levels of VEGF were measured. Data are shown as mean $\pm \mathrm{SEM} ; \mathrm{n}=4$. $^{* * *} \mathrm{P}<0.001$. Flu, fluvastatin; Nor, normoxia; Hyp, hypoxia.

Inhibition of VEGF caused by fluvastatin under hypoxia. VEGF is considered as a highly specific mitogen for endothelial cells and a common biological biomarker of angiogenesis (13). To investigate the role of statins on VEGF expression under hypoxia, we examined VEGF under conditions of normoxia and hypoxia with or without fluvastatin. Hypoxia increased the mRNA and protein expression of VEGF, however, VEGF level was dramatically decreased to normoxic level after fluvastatin treatment (Fig. 6).

\section{Discussion}

Endothelial cells exhibit an immense proliferative activity response to hypoxia. Hypoxic cells can maintain energy generation and biomolecule production to survive and even proliferate through adjusting the way of glucose (14) and glutamine metabolisms (15) in the adaptation process. Moreover, a number of mitogenic factors and pathways are activated to sustain the proliferation phenotype in endothelial cells, including VEGF, endothelin-1, PDGFB, IGFBP3, placental growth factor (PGF) (16) and mammalian target of rapamycin (mTOR) signaling (17). In the present study, hypoxia exerted a prominent proliferative effect on HUVEC by regulating $G_{1} / S$ transition.

Statins, as the first-line agents in hypercholesteremia therapy, have benefits in hypertensive patients and are able to lower the risk of developing cardiovascular events (18). Since hypertension is a common complication of traditional anti-angiogenic therapy in patients with cancer (19), the supply of statins is an attractive strategy in cancer treatment. Plenty of preclinical studies have revealed that the combination of statins and other chemotherapeutics enhance the effectiveness of cancer treatment (20). Long-term use of statins for more than one year is proven to be associated with a decreased risk of endometrial cancer (21) and a $36 \%$ reduction of colorectal cancer-specific mortality (22).
Our research demonstrated that statins inhibited cell growth by inducing cell apoptosis and regulating cell cycle under hypoxia in a dose- and time-dependent manner. Previously it was reported that statins block hypoxia-induced endothelial proliferation through decreasing $\mathrm{Ca}^{2+}$ and preventing ROS generation (23). However, the underlying molecular mechanisms are poorly understood.

Statins block the synthesis of mevalonate by competitively inhibiting $\mathrm{HMG}-\mathrm{CoA}$ reductase. Mevalonate is a precursor of coenzyme Q10, a component which plays a key role in mitochondrial respiration and facilitates mitochondrial bioenergy transfer (24). Inhibition of the mevalonate pathway engaged by statin is shown to disrupt mitochondrial surveillance (25). The results of antibody array revealed the involvement of bcl-2 and caspase-3, implying that mitochondrial pathway may be implicated in the regulation of cell growth by fluvastatin. Therefore, we performed qPCR and western blot experiments to test the hypothesis. As expected, data showed that fluvastatin significantly increased bax/bcl-2 ratio and triggered release of cytochrome $c$ from mitochondria and then induced the cleavage of caspase-3, caspase- 9 and eventually PARP.

Cytochrome $c$ is able to transfer electrons between cytochrome complexes III and IV, in order to maintain proper energy conversion. Mitochondrial dysfunction caused by mitochondrial complex III inhibitor significantly suppresses the hypoxia-induced VEGF expression in human proximal tubular epithelial cells (hPTECs) (26). Based on the central role of VEGF in hypoxic cell proliferation, we measured the expression of VEGF in HUVEC with fluvastatin or DMSO treatment. Consistent with the study of Loboda et al (27) in human microvascular endothelial cells (HMEC-1), our results suggested that VEGF was augmented under hypoxia and attenuated to normoxic level following fluvastatin treatment. However, the underlying association of VEGF and mitochondrial apoptosis warrants further investigation. 
Fluvastatin was revealed to prevent endothelial cells from leaving $\mathrm{G}_{1}(9)$ and cause $\mathrm{G}_{2} / \mathrm{M}$ arrest in leukemic natural killer cells (28). In the present study, fluvastatin induced the accumulation of $\mathrm{G}_{0}-\mathrm{G}_{1}$ phase cells and correspondingly reduced the proportion of cells in $\mathrm{S}$ phase time-dependently, slowing down the progression of cell mitosis.

The altered expression was confirmed in hypoxic HUVEC treated with fluvastatin or DMSO, including cell cycle regulators IGFBP-6, cyclin B1, cyclin D1, p27, p53, as well as proliferation-related protein survivin. IGFBP-6 was identified as oncosuppressor in nasopharyngeal carcinoma (29) and inhibits cell proliferation in connective tissue disease (30). Cells overexpressing IGFBP- 6 are blocked at the $G_{1} / S$ transition compared with scramble cells (31). Cyclin B1 and cyclin D1 are critical cell cycle regulatory proteins. Cyclin B1 mRNA and protein levels were strongly inhibited following fluvastatin treatment while $\mathrm{G}_{2}-\mathrm{M}$ phase cells were not accumulated. Cell cycle checkpoints arrest in response to DNA damage contributes to allow time for DNA repair and defects in the $\mathrm{G}_{2}-\mathrm{M}$ arrest are associated with damaged cell mitosis and final cell death (32), which may help explain the above described results. P27 and p53 are referred as tumor suppressors because of their major function on cell cycle inhibition $(33,34)$. P27 negatively regulates $G_{1}$ progression and strongly interacts with cyclin D1. P53 controls both the $G_{1}$ and $G_{2}$ checkpoints and also mediates cell apoptosis in response to DNA damage through transcriptionally activating bax and repressing bcl-2 (35). Survivin is highly expressed in the majority of carcinomas and has an important bearing on cell apoptosis and proliferation (36). DNA damage is defined to be related with p53-survivin signaling pathway to induce cell apoptosis and cell cycle arrest (37), suggesting that a potential cross talk between different signaling pathways existed in the effect of statins on HUVEC.

The above study is a protein array-based screen for specific proteins involved in the process. However, the expression for some proteins in the apoptosis array analysis is not consistent with the results from qPCR and western blot experiments, such as bax and p53. The discrepancy may be attributed to a tendency to generate false negative signals in a high-throughput method (38).

This is the first report comprehensively describing the mechanism underlying the effect of statins on vascular endothelial cell survival under hypoxia. Our data revealed that fluvastatin induced apoptosis through mitochondrial pathway and inhibited cell proliferation by increasing IGFBP-6, p27, p53 and decreasing cyclin B1, cyclin D1, survivin, VEGF levels. Statin treatment may have potential for effective cancer therapy.

\section{Acknowledgements}

The present study was supported by grants from the Natural Science Foundation of China (81270106), the Ministry of Science and Technology of China (2012BAI05B00) and the Ministry of Public Health (201002008).

\section{References}

1. Nishida N, Yano H, Nishida T, Kamura T and Kojiro M: Angiogenesis in cancer. Vasc Health Risk Manag 2: 213-219, 2006.
2. Uzzan B, Nicolas P, Cucherat M and Perret GY: Microvessel density as a prognostic factor in women with breast cancer: a systematic review of the literature and meta-analysis. Cancer Res 64: 2941-2955, 2004

3. Bremnes RM, Camps C and Sirera R: Angiogenesis in non-small cell lung cancer: the prognostic impact of neoangiogenesis and the cytokines VEGF and bFGF in tumours and blood. Lung Cancer 51: 143-158, 2006.

4. Zhao HC, Qin R, Chen XX, et al: Microvessel density is a prognostic marker of human gastric cancer. World J Gastroenterol 12: 7598-7603, 2006

5. Vaupel P: The role of hypoxia-induced factors in tumor progression. Oncologist 9 (Suppl 5): S10-S17, 2004.

6. Fang L, Choi SH, Baek JS, et al: Control of angiogenesis by AIBP-mediated cholesterol efflux. Nature 498: 118-122, 2013

7. Wang CY, Liu PY and Liao JK: Pleiotropic effects of statin therapy: molecular mechanisms and clinical results. Trends Mol Med 14: 37-44, 2008.

8. Grosser N, Hemmerle A, Berndt G, et al: The antioxidant defense protein heme oxygenase 1 is a novel target for statins in endothelial cells. Free Radic Biol Med 37: 2064-2071, 2004.

9. Newton CJ, Ran G, Xie YX, et al: Statin-induced apoptosis of vascular endothelial cells is blocked by dexamethasone. J Endocrinol 174: 7-16, 2002.

10. Asakage M, Tsuno NH, Kitayama J, et al: 3-Hydroxy-3methylglutaryl-coenzyme A reductase inhibitor (pravastatin) inhibits endothelial cell proliferation dependent on G1 cell cycle arrest. Anticancer Drugs 15: 625-632, 2004.

11. Weis M, Heeschen C, Glassford AJ and Cooke JP: Statins have biphasic effects on angiogenesis. Circulation 105: 739-745, 2002.

12. Gross A, McDonnell JM and Korsmeyer SJ: BCL-2 family members and the mitochondria in apoptosis. Genes Dev 13: 1899-1911, 1999.

13. Hoeben A,Landuyt B, Highley MS, Wildiers H, Van Oosterom AT and De Bruijn EA: Vascular endothelial growth factor and angiogenesis. Pharmacol Rev 56: 549-580, 2004.

14. Polet F and Feron O: Endothelial cell metabolism and tumour angiogenesis: glucose and glutamine as essential fuels and lactate as the driving force. J Intern Med 273: 156-165, 2013.

15. Wise DR, Ward PS, Shay JE, et al: Hypoxia promotes isocitrate dehydrogenase-dependent carboxylation of alpha-ketoglutarate to citrate to support cell growth and viability. Proc Natl Acad Sci USA 108: 19611-19616, 2011.

16. Manalo DJ, Rowan A, Lavoie T, et al: Transcriptional regulation of vascular endothelial cell responses to hypoxia by HIF-1. Blood 105: 659-669, 2005.

17. Humar R, Kiefer FN, Berns H, Resink TJ and Battegay EJ: Hypoxia enhances vascular cell proliferation and angiogenesis in vitro via rapamycin (mTOR)-dependent signaling. FASEB J 16: 771-780, 2002.

18. Osende JI, Ruiz-Ortega M, Blanco-Colio LM and Egido J: Statins to prevent cardiovascular events in hypertensive patients. The ASCOT-LLA study. Nephrol Dial Transplant 19: 528-531, 2004.

19. Pande A, Lombardo J, Spangenthal E and Javle M: Hypertension secondary to anti-angiogenic therapy: experience with bevacizumab. Anticancer Res 27: 3465-3470, 2007.

20. Osmak M: Statins and cancer: current and future prospects. Cancer Lett 324: 1-12, 2012.

21. Lavie O, Pinchev M, Rennert HS, Segev Y and Rennert G: The effect of statins on risk and survival of gynecological malignancies. Gynecol Oncol 130: 615-619, 2013.

22. Cardwell CR, Hicks BM, Hughes C and Murray LJ: Statin use after colorectal cancer diagnosis and survival: a populationbased cohort study. J Clin Oncol 32: 3177-3183, 2014.

23. Schaefer CA, Kuhlmann CR, Weiterer S, et al: Statins inhibit hypoxia-induced endothelial proliferation by preventing calciuminduced ROS formation. Atherosclerosis 185: 290-296, 2006.

24. Deichmann R, Lavie C and Andrews S: Coenzyme q10 and statininduced mitochondrial dysfunction. Ochsner J 10: 16-21, 2010.

25. Liu Y, Samuel BS, Breen PC and Ruvkun G: Caenorhabditis elegans pathways that surveil and defend mitochondria. Nature 508: 406-410, 2014.

26. Satoh M, Fujimoto S, Horike H, et al: Mitochondrial damageinduced impairment of angiogenesis in the aging rat kidney. Lab Invest 91: 190-202, 2011.

27. Loboda A, Jazwa A, Jozkowicz A, Molema G and Dulak J: Angiogenic transcriptome of human microvascular endothelial cells: effect of hypoxia, modulation by atorvastatin. Vascul Pharmacol 44: 206-214, 2006. 
28. Crosbie J, Magnussen M, Dornbier R, Iannone A and Steele TA: Statins inhibit proliferation and cytotoxicity of a human leukemic natural killer cell line. Biomark Res 1: 33, 2013.

29. Kuo YS, Tang YB, Lu TY, Wu HC and Lin CT: IGFBP-6 plays a role as an oncosuppressor gene in NPC pathogenesis through regulating EGR-1 expression. J Pathol 222: 299-309, 2010.

30. Raykha C, Crawford J, Gan BS, Fu P, Bach LA and O'Gorman DB: IGF-II and IGFBP-6 regulate cellular contractility and proliferation in Dupuytren's disease. Biochim Biophys Acta 1832: 1511-1519, 2013.

31. Grellier P, De Galle B and Babajko S: Expression of insulin-like growth factor-binding protein 6 complementary DNA alters neuroblastoma cell growth. Cancer Res 58: 1670-1676, 1998.

32. DiPaola RS: To arrest or not to $\mathrm{G}_{2}-\mathrm{M}$ Cell-cycle arrest: commentary re: A.K. Tyagi, et al: Silibinin strongly synergizes human prostate carcinoma DU145 cells to doxorubicin-induced growth inhibition, $\mathrm{G}_{2}-\mathrm{M}$ arrest, and apoptosis. Clin Cancer Res 8: 3512-3519, 2002. Clin Cancer Res 8: 3311-3314, 2002.
33. Toyoshima $\mathrm{H}$ and Hunter T: p27, a novel inhibitor of G1 cyclinCdk protein kinase activity, is related to p21. Cell 78: 67-74, 1994.

34. Yin Y, Tainsky MA, Bischoff FZ, Strong LC and Wahl GM: Wild-type p53 restores cell cycle control and inhibits gene amplification in cells with mutant p53 alleles. Cell 70: 937-948, 1992.

35. Basu A and Haldar S: The relationship between BcI2, Bax and p53: consequences for cell cycle progression and cell death. Mol Hum Reprod 4: 1099-1109, 1998.

36. Sarela AI, Verbeke CS, Ramsdale J, Davies CL, Markham AF and Guillou PJ: Expression of survivin, a novel inhibitor of apoptosis and cell cycle regulatory protein, in pancreatic adenocarcinoma. Br J Cancer 86: 886-892, 2002.

37. Zhou M, Gu L, Li F, Zhu Y, Woods WG and Findley HW: DNA damage induces a novel p53-survivin signaling pathway regulating cell cycle and apoptosis in acute lymphoblastic leukemia cells. J Pharmacol Exp Ther 303: 124-131, 2002.

38. Maslov S and Sneppen K: Protein interaction networks beyond artifacts. FEBS Lett 530: 255-256, 2002. 